\title{
Addressing the Deployment Challenges of Health Monitoring Devices for a Dementia Study
}

\author{
T. Collins \\ S. I. Woolley \\ School of Electronic, Electrical and Systems \\ Engineering \\ University of Birmingham \\ Edgbaston, Birmingham, UK. \\ t.collins@bham.ac.uk \\ s.i.woolley@bham.ac.uk
}

\author{
S. Aldred \\ S. Rai \\ School of Sport, Exercise and Rehabilitation \\ Sciences \\ University of Birmingham \\ Edgbaston, Birmingham, UK. \\ s.aldred.1@bham.ac.uk \\ SXR294@student.bham.ac.uk
}

\begin{abstract}
This paper presents the findings of a technological adoption assessment of health monitoring devices for a dementia study. The work was motivated by the need to monitor physical activity interventions in a study cohort of dementia patients living with caregiver support in the community. The system requirements were for a discrete and unobtrusive solution with activity level (energy expenditure) and heart rate monitoring. In addition to fulfilling system requirements, successful technology adoption requires careful consideration of practical challenges in deployment. The paper addresses these challenges - in particular, aspects relating to the servicing and maintenance of units over the study period and the access and synchronisation of data. Test data visualisations and data mining results for sustained, long-term data capture are provided.
\end{abstract}

\section{Categories and Subject Descriptors}

J.3 [Life and Medical Sciences]: Health; J.3 [Life and Medical Sciences]: Medical information systems

\section{General Terms}

Health Monitoring, Activity Monitoring

\section{Keywords}

Activity Monitoring, Dementia

\section{INTRODUCTION}

Dementia is a term used to describe several neurodegenerative disorders, of which Alzheimer's disease is the most common, affecting millions worldwide. Physical activity and exercise are known to reduce risks for diseases such as Alzheimer's disease, cardiovascular disease and diabetes by decreasing oxidative stress and inflammation in the periphery [10]. Evidence from animal-based research has shown that exercise promotes extensive vascular changes and adaptive mechanisms in the central nervous system (e.g. growth of blood vessels), consequently improving brain blood flow. However, whilst the most effective exercise has arguably been characterised for prevention and treatment of cardiovascular complaints, little is known about the best form of exercise to benefit people with dementia.

Monitoring the levels of physical activity in people with dementia is not straightforward. Dementia sufferers are anxious about change [5] and they can become very agitated by anything new appearing in the home, or on the person [12]. In addition, the loss of short-term memory can make simple solutions, such as the compilation of an activity log, unsuitable. A wearable, self-contained, solution is preferable. A 2012 review of wearable and ambient sensing systems for health and rehabilitation highlighted the potential of objective monitoring for home-based rehabilitation interventions [9]. In addressing dementia, the authors observed that an "important factor in this patient population is that the monitoring system must be totally unobtrusive and, if possible, collect information in a transparent way without patient intervention".

While research effort in remote and "sensor-less" sensing (opportunistic sensing from devices in daily use) [8] offers some potential in terms of future solutions meeting the twin ideal of totally unobtrusive and nothing new, the recent surge in commercially available solutions for wearable activity and health monitoring devices evidences moves in the right direction. In this paper we explore and compare candidate products closest to this ideal and which comply with the study requirements. The practical issues surrounding implementation are reported together with test results for sustained, long-term data capture with visualisation analytics from raw data and data mining.

\section{SYSTEM REQUIREMENTS}

Study requirements in terms of system sensing, data access and invasiveness were determined. The study design was for a 4 -week physical activity intervention for a patient cohort with a moderate degree of dementia living with caregiver 
Table 1: Comparison of Monitoring Systems

\begin{tabular}{|l|c|c|c|c|c|c|}
\hline $\begin{array}{l}\text { Device } \\
\text { Manufacturer - Product }\end{array}$ & $\begin{array}{c}\text { Activity } \\
\text { Monitoring }\end{array}$ & $\begin{array}{c}\text { Pulse } \\
\text { Monitoring }\end{array}$ & $\begin{array}{c}\text { EDA } \\
\text { Monitoring }\end{array}$ & $\begin{array}{c}\text { Other } \\
\text { Sensors }\end{array}$ & $\begin{array}{c}\text { Raw Data } \\
\text { Access }\end{array}$ & Price \\
\hline Basis - Peak & Yes & Yes & Yes & Skin Temp. & Yes & $£ 200$ \\
\hline Polar - FT60 & Yes & Yes & No & GPS & Yes & $£ 160$ \\
\hline Mio - Alpha & No & Yes & No & - & Yes & $£ 150$ \\
\hline Jawbone - Up24 & Yes & No & No & - & No $^{* *}$ & $£ 100$ \\
\hline Withings - Pulse Ox & Yes & Yes & No & SpO $_{2}^{* * *}$ & Yes & $£ 100$ \\
\hline Nike - Fuelband & Yes & No & No & - & No $^{* *}$ & $£ 90$ \\
\hline Fitbit - Flex & Yes & No & No & - & Yes & $£ 80$ \\
\hline
\end{tabular}

* Requires separate chest-strap sensor for pulse rate measurement

** Raw data access is not supported by the manufacturer but 'hacks' to achieve it are reported online

*** $\mathrm{SpO}_{2}=$ Peripheral capillary oxygen saturation (pulse oximetry)

support in their own homes. Up to 30 study participants could require concurrent monitoring over 4 weeks. Device monitoring was provisioned to supplement other study data including health, behaviour and quality of life assessments.

\subsection{Sensors}

Activity monitoring is attempted to some degree by even the most basic of commercial devices and can also be achieved, in part, via embedded smartphone accelerometers. However, to better inform the assessment of energy expenditure, additional measures such as heart rate and electrodermal activity (EDA) (an indicator of perspiration) were desirable. In combination with accelerometer data, these metrics allow different types and intensities of physical activity to be characterised. GPS location data was also desirable but not essential.

\subsection{Access to Raw Data}

Access to raw data may seem an obvious requirement but its support cannot be assumed. A number of systems use proprietary communication protocols to relay sensor data to a smartphone or PC and only provide access to summarised data rather than the full set of recorded sensor data. We planned to analyse and mine the data and therefore required access to the raw sensor data, for example, in CSV (CommaSeparated Value) or XML (Extensible Markup Language) form.

\subsection{Invasiveness}

Systems with separate sensors that attach to different parts of the body can give good results but were deemed too invasive for the dementia study cohort. Expert advice recommended that less invasive, single unit, belt-worn devices and wrist-watch formats were preferred.

\section{COMPARISON OF MONITORING SYSTEMS}

ACTIVITY

A survey of commercial activity monitoring systems was conducted in December 2014. The candidate devices, summarised in table 1, were: Fitbit Flex [2]; Nike+ Fuelband SE [7]; Jawbone Up [4]; Withings Pulse Ox [13]; Mio Alpha [6]; Polar FT60 [11]; and Basis Peak [1]. All are wrist watch formats with the exception of Withings Pulse Ox which can optionally be belt-worn.

The activity monitoring market is evolving very quickly and within six months of the survey, several models had already been superseded. For example, newer devices from Fitbit, Mio, Jawbone and Microsoft include pulse monitoring and GPS. These devices would be good candidates for future studies. At the time of our survey, however, these models were not available.

Devices that did not measure pulse rate were eliminated first. The Withings' Pulse Ox can measure pulse rate but not continually. To measure pulse the wearer removes the device from their wrist and puts their finger over an optical sensor on the back. This requirement was deemed unsuited to the study participants. The Mio Alpha was eliminated because it did not provide activity monitoring data. The remaining contenders were the Polar FT60 and the Basis Peak. Although the Polar FT60 provided GPS sensing, it was eliminated because of the practicalities of the pulse sensor. Unlike the other devices which use optical sensors to detect pulse rate, the FT60 required a separate heart-rate sensor mounted on a chest strap. The Basis Peak, made available in the UK in January 2015, was the only remaining device that met the study requirements and also provided additional skin temperature data.

\section{DEPLOYMENT CHALLENGES}

Study adoption required the technology to be deployed with up to thirty participants concurrently for four weeks. To reduce participant invasion and researcher workload, the preferred number of researcher visits to participant homes was limited to one per week.

\subsection{Battery Life}

Limited battery life is a common problem associated with wearable computing devices. The Basis Peak watch has a battery life of between 3-5 days depending on usage. Wireless data synchronisation, for example, is an energy-demanding function. With only one researcher home visit per week, there was a need for additional recharging. It was determined that placing the watch in its charging cradle for ten minutes each day would maintain the charge and could be adopted into caregiver responsibilities.

\subsection{Data Synchronisation}

Data synchronisation presented something of a challenge. The watch is designed to synchronise data with an iPhone 

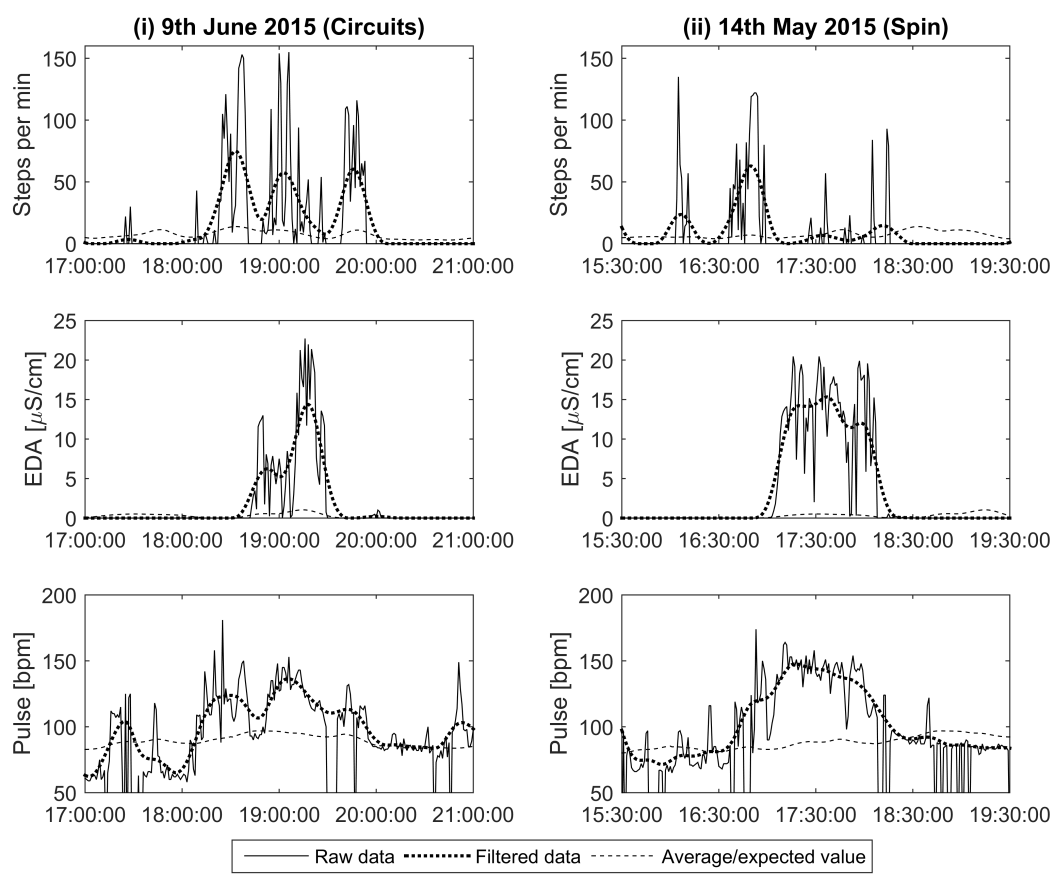

Figure 1: Examples of steps-per-minute, EDA and pulse rate data collected during two four-hour periods during testing.

(iOS) or Android smartphone via a wireless Bluetooth connection using the Basis app which communicates via the Internet with the database held by Basis.

There were initial problems with Android phone use because the Bluetooth interface was not compatible with the latest version of the operating system (v5). However, using an older phone (with Android v4.4) and disabling system updates provided a workaround. No similar problems were encountered with iOS devices.

\subsection{Deploying Multiple Units}

Although slightly convoluted, the process of data acquisition worked satisfactorily with a single watch. In the study, however, thirty watches would be deployed simultaneously. Each watch expects to pair with a phone but the current phone app does not support multiple watches or multiple users. In order to synchronise data from more than one watch with a single phone, it is necessary to first create separate user accounts for the different watches. Then to synchronise, the phone and watch must be paired and the account details entered into the app. Once synchronisation is complete, the account details must be deleted and the watch unpaired in order to repeat the process with another device. This is not a procedure that one would wish to repeat daily. The watch is capable, however, of buffering at least a week's worth of data - although the time taken to upload this via the Bluetooth link can be quite lengthy.

A simple solution to the multiple unit problem would be to provision a phone for every participant. This increases the equipment costs and is wasteful given that the phone's capabilities would otherwise not be required. Single-board computers running Android OS were considered for an alternative solution. The most cost-effective options being media-streaming Android boxes which, at the time of writing, can be sourced for as little as $£ 20$. These devices require an HDMI monitor to set-up initially, but can then be left to operate without a display and will automatically upload data from the watch to the Internet at regular intervals.

\section{PILOT TESTING AND VISUAL ANALYTICS}

As part of the technological pilot testing, researchers wore the Basis Peak watches for periods of several days and weeks. Reliability is a common problem with ambulatory sensing especially with sensors requiring skin contact: pulse rate, EDA and skin temperature in this case. However, analysis of the raw sensor data revealed fairly good performance with $90 \%$ of sensor losses lasting less than five minutes. For the purposes of study monitoring, it was judged that this missing data could be approximated by interpolation. Data recorded during different examples of fairly intense activity are plotted in figure 1 and show the result of this processing. Raw data for both EDA and pulse rate exhibits several short losses. The filtered data was processed by averaging valid samples within a cosine-squared windowed block of 30 minutes duration. Also shown in figure 1 is the 'expected' value of each parameter calculated by averaging the readings from each of the 45 days during the testing period. The data in figure 1(i) was gathered during a circuit training exercise class and, as shown, the steps per minute, EDA and pulse are significantly higher than average. In this example, physical activity could have been detected from the pedometry data alone. However, figure 1(ii) shows data collected from a spin (indoor cycling) session. In this case, the wrist remains virtually static so the accelerometer senses very low activity. 


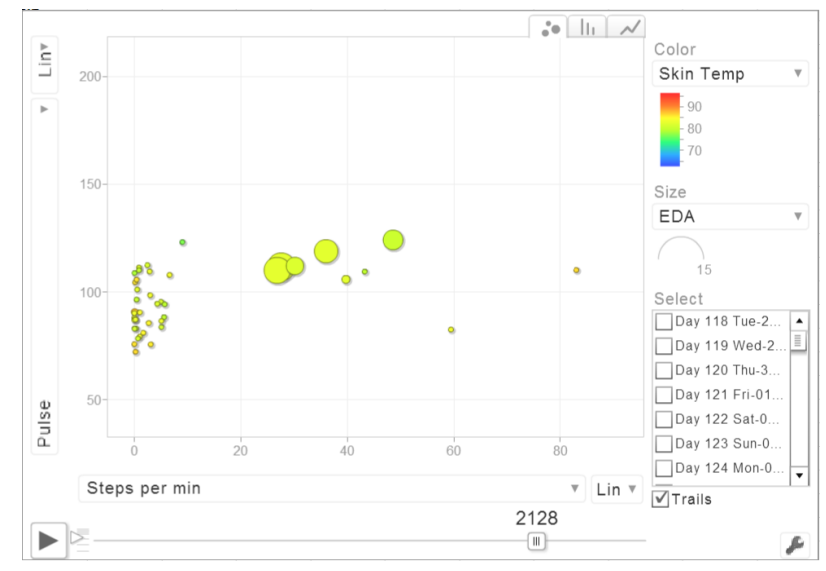

Figure 2: Example of motion chart visualisation of 45 days of data.

It is only the EDA and pulse data that show evidence of significant physical exertion.

Relationships between the multiple sensed parameters were visualised using Google's Motion Chart [3] dynamic visualisation tool. Figure 2 shows a snapshot. Each 'bubble' represents data from a different day, bubble size varies according to EDA and colour according to skin temperature. Animating over the course of 24 hours reveals patterns of behaviour that can be difficult to visualise by other means.

Data mining techniques were used to characterise activities. The example scatter plot in figure 3 shows the results of $k$-means clustering. Here the data has mostly been segregated across the pulse/skin-temperature plane. Cluster C6, however, appears to overlap with several other clusters but, in fact, is differentiated by a higher steps-per-minute parameter.

\section{CONCLUSIONS}

Careful device selection decisions, including timely consideration of practical implementation issues, are important to the design of monitoring studies. With devices evolving very quickly there is, necessarily, a new improved option near-market or another just arrived but too late for adoption. Waiting for the ideal device could take years and, with crises in dementia care from the scale of demands in our aging societies, there is a need to make the best of currently available technology. Careful assessment of deployment challenges has the potential to contribute by substantially reducing obstacles to effective study adoption of new technologies. In addition, pilot testing and data analysis can provide a degree of confidence in terms of system robustness and data processing requirements with visual analytics and data mining providing insights into the substance of sensed recordings.

\section{ACKNOWLEDGMENTS}

This work was funded, in part, by the European Union through a European Regional Development Fund - Accelerating Business Knowledge Base Activity award in collaboration with Care Companions Ltd.

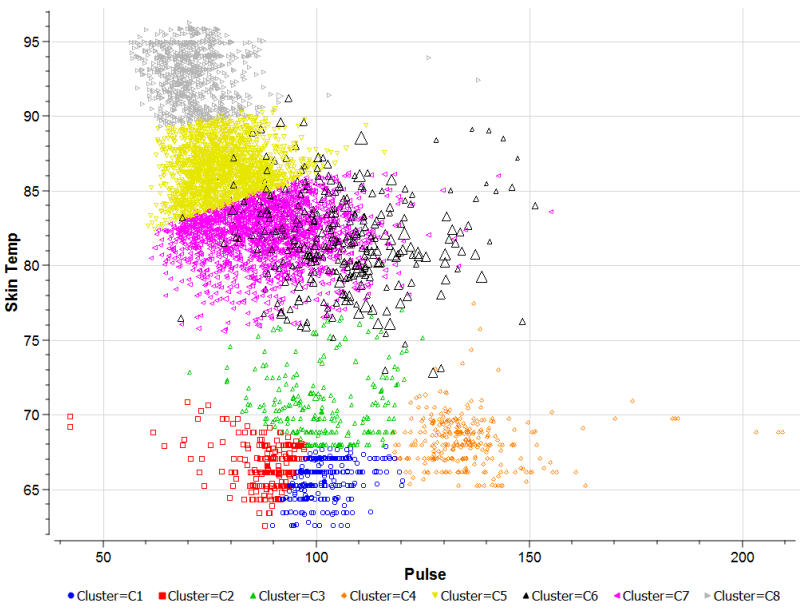

Figure 3: Results from $k$-means clustering of 45 days of data. (Point colours correspond to clusters C1-8; point size corresponds to steps-per-minute.)

\section{REFERENCES}

[1] Basis. Basis Peak, 2014. http://en-gb.mybasis.com/ [accessed 10-Dec-2014].

[2] Fitbit. Fitbit Flex, 2014. https://www.fitbit.com/uk/flex [accessed 10-Dec-2014].

[3] Google. Motion charts, 2014. https://developers.google.com/chart/ [accessed 17-Jun-2015].

[4] Jawbone. Jawbone Up, 2014. https://jawbone.com/up [accessed 10-Dec-2014].

[5] M. S. Mega, J. L. Cummings, T. Fiorello, and J. Gornbein. The spectrum of behavioral changes in alzheimer's disease. Neurology, 46(1):130-135, 1996.

[6] Mio. Mio Alpha, 2014. http://www.mioglobal.com/ [accessed 10-Dec-2014].

[7] Nike. Nike+ Fuelband SE, 2014. http://store.nike.com [accessed 10-Dec-2014].

[8] P. Paredes, D. Sun, and J. Canny. Sensor-less sensing for affective computing and stress management technology. In Pervasive Computing Technologies for Healthcare (PervasiveHealth), 2013 7th International Conference on, pages 459-463, May 2013.

[9] S. Patel, H. Park, P. Bonato, L. Chan, M. Rodgers, et al. A review of wearable sensors and systems with application in rehabilitation. J Neuroeng Rehabil, 9(12):1-17, 2012

[10] A. M. W. Petersen and B. K. Pedersen. The anti-inflammatory effect of exercise. Journal of applied physiology, 98(4):1154-1162, 2005.

[11] Polar. Polar FT60, 2014. http://www.polar.com/ [accessed 10-Dec-2014].

[12] H. Ragneskog, L. A. Gerdner, K. Josefsson, and M. Kihlgren. Probable reasons for expressed agitation in persons with dementia. Clinical Nursing Research, 7(2):189-206, 1998.

[13] Withings. Withings Pulse Ox, 2014. http://www2.withings.com [accessed 10-Dec-2014]. 